\title{
Acquisition de la phonologie du français : Enjeux théoriques et méthodologiques, données
}

\author{
Sophie Wauquier \\ Structure formelle du langage : typologie et acquisition, metrique et poétique (SFLTAMP) \\ sophie.wauquier@orange.fr
}

La phonologie du français a longtemps été examinée, analysée et formalisée exclusivement sur les données d'adultes. Les champs de l'acquisition phonologique et plus généralement de la psycholinguistique de la parole se sont ouverts en France à partir des années 90.

Nous ferons l'état des lieux quasiment vingt ans après, en nous centrant sur les points suivants.

\section{Corpus et méthodes}

Des données de français (Grégoire, Boysson-Bardies, Vihman, Veneziano, Le Normand) existaient fragmentairement et de manière éparpillée. Depuis les années 2000, deux tendances se sont affirmées : la constitution de corpus (notamment des corpus longitudinaux) plus systématiques et plus volumineux et la mise à disposition de ces corpus via Childes et Phon (Y. Rose) pour la communauté scientifique. A ceci s'ajoute le développement d'une articulation féconde entre psycholinguistique expérimentale et phonologie, complétant les analyses que l'on peut dégager de l'observation des données.

\section{Objets}

Les problèmes spécifiques que pose la phonologie du français (liaison, schwa, voyelles moyennes, variations diatopiques) ont été une mine d'inspiration pour la phonologie adulte des années 70 . Certaines thématiques sont maintenant l'objet de travaux attestés en l'acquisition (la liaison, la variation diatopique). D’autres (comme le schwa) restent à défricher.

Par ailleurs, les thématiques classiques observés pour les langues germaniques (taille et forme de la première unité, acquisition segmentale et marque, acquisition de la syllabe, harmonie consonantique, lien entre acquisition segmentale et supra segmentale, interface morphologie-phonologie) commencent à être productives pour le français.

\section{Enjeux théoriques}

Les enjeux théoriques sont de triple nature.

Les résultats et analyses visent d'une part la compréhension des mécanismes d'apprentissage et d'acquisition de la phonologie du français et par là de toute phonologie. L'absence de récursivité, le caractère fini du nombre d'unités à acquérir ainsi que la dimension systémique de la phonologie interrogent sous un angle particulier les théories de l'acquisition et de l'apprentissage (innéistes, émergentistes, fonctionnalistes etc ...)

Les résultats et analyses visent également à renseigner la connaissance que nous avons de la phonologie du français via les contraintes qu'elle impose à l'apprentissage. De ce point de vue, on soulèvera les questions liées au problème de la continuité entre les théories phonologiques rendant compte du français chez l'adulte et les propositions pour l'enfant. 
Enfin, les résultats et analyses peuvent s'envisager sous un angle typologique. L'acquisition de la phonologie a été bien étudiée pour les langues germaniques (anglais, allemand, néerlandais). Beaucoup moins pour les langues romanes. Les travaux sur le français apportent un éclairage neuf sur les objets et élargissent le champ des questions posées. Ils permettent en outre d'enrichir la base empirique sur laquelle s'appuient les débats concernant l'universalité des systèmes et de leur mode d'acquisition. 